\title{
Genetic circuit design automation for yeast
}

Ye Chen $^{1 \dagger}$, Shuyi Zhang ${ }^{1}$, Eric M. Young ${ }^{1}$, and Christopher Voigt ${ }^{1,2^{*}}$

${ }^{1}$ Synthetic Biology Center, Department of Biological Engineering, Massachusetts Institute of Technology, Cambridge, MA, USA

†Present address: CAS Key Laboratory of Quantitative Engineering Biology, Shenzhen Institute of Synthetic Biology, Shenzhen Institutes of Advanced Technology, Chinese Academy of Sciences, Shenzhen 518055, China

*Corresponding and request for materials should be addressed to C.A.V. (cavoigt@gmail.com)

Key words: $\quad$ Synthetic Biology, Systems Biology, Electronic Design Automation, Fermentation 


\begin{abstract}
Cells can be programmed to monitor and react to their environment using genetic circuits. Design automation software maps a desired circuit function to a DNA sequence, which requires units of gene regulation (gates) that are simple to connect and behave predictably. This poses a challenge for eukaryotes due to their complex mechanisms of transcription and translation. To this end, we have developed gates for yeast (Saccharomyces cerevisiae) that are connected using RNA polymerase (RNAP) flux as the signal carrier and are insulated from each other and host regulation. They are based on minimal constitutive promoters ( $120 \mathrm{bp})$, for which rules are developed to insert operators for DNA-binding proteins. Using this approach, we construct nine NOT/NOR gates with nearly-identical response functions and 400-fold dynamic range. In circuits, they are transcriptionally insulated from each other by placing ribozymes downstream of terminators to block nuclear export of mRNAs resulting from RNAP readthrough. Based on these gates, Cello is used to build circuits to specification with up to 11 regulatory proteins. A simple dynamic model predicts the circuit response over days. Extending genetic circuit design automation to eukaryotes simplifies their incorporation into cellular engineering projects, whether it be to stage processes for bioproduction, serve as environmental sentinels, or guide living therapeutics.
\end{abstract}




\section{Introduction}

When engineering yeast, it would be powerful to be able to program cells to do different things at different times. For example, they could create hierarchal structures as they grow into living materials or work together during fermentation to build a complex product [1-6]. Progress has been made in building circuits in Saccharomyces cerevisiae, including logic gates and memory [1, 7-22]. However, building such circuits requires specialized expertise and, while often qualitatively guided by mathematical models, the mapping of a function to a DNA sequence is a qualitative and slow trial-and-error process $[1,7,23-26]$. Cello is design automation software that seeks to empower engineers to incorporate circuits into their projects, while abstracting them from the details of designing the DNA sequence. A user specifies the circuit function in a species-independent manner using Verilog and then this is mapped by the software to a particular organism using data in a "user constraint file" (UCF) (Fig. 1). The UCF also defines the location of the circuit, the gates and mathematical model used to connect them, and composition rules for the DNA sequence. To date, UCFs have been built for the prokaryotes Escherichia coli DH10 $\beta$ ( $15 a$ plasmid) and Bacteroides thetaumicron (genome), but have not been developed for a eukaryote[27-29].

NOR gates can be connected to build any circuit and the biochemistry required to construct them is simple $[24,30,31]$. They have two input promoters that express a repressor that turns off an output promoter. Thus, the signal connecting the gates is the RNAP flux emanating from the promoters. The response function of a gate captures how the output changes as the function of the inputs(s) and is used by mathematical models to predict how gates can be combined to build a circuit [27]. Measuring RNAP flux is difficult, so the promoter activities are compared to a reference promoter and reported in "relative promoter units" (RPU) [32, 33].

The precision of design automation software is only as good as the gate technology. Gates must generate the same response in the context of different circuits. For prokaryotic gates, this has been achieved using insulators, including ribozymes, long promoters, and strong terminators [25, 34-38]. Eukaryotic regulation is more complex and difficult to insulate. Promoter strength can be impacted by global chromosome structure, nucleosome occupancy, pervasive transcription, and interactions with the nuclear pore complex [39-46]. Chromosomes have natural mechanisms of insulation, for example to block silencers or the spread of heterochromatin, but their function cannot be reduced to a discrete genetic part [47-50]. Native promoters have been used as scaffolds to introduce operators for DNA-binding regulatory proteins, but they often remain sensitive to environmental and genetic context $[1,42,47,51$ 55]. To address these issues, minimal promoters have been designed [42, 52, 56-58]. Neighboring 
transcription units can also influence each other by affecting nucleosome repositioning [59-62]. It has been found that T-rich promoters lead to nucleosome-free regions, thus leading to strong promoters with reduced noise that are less impacted by neighboring DNA $[40,57,62-66]$. Promoters also can be impacted by the downstream terminator because it can re-initiate RNAP [46, 57, 67-75]. This can also make the use of terminators to separate transcription units problematic $[76,77]$. To insulate against chromatin structure, Klavins and co-workers developed CRISPRi-based gates where dCas9 is fused to a chromatin remodeling domain [24].

We have designed short ( $<120 \mathrm{bp})$ synthetic promoters that can be strongly repressed and use these to build three sensors and nine NOT/NOR gates based on prokaryotic DNA-binding proteins. Rules for operator placement are developed based on a 20-T spacer that is diversified to avoid homologous recombination. The gates produce near-identical response functions with up to 100-fold dynamic range and low basal transcription. The gate thresholds are tuned to be similar through the selection of Kozak sequences. The gates are integrated into the chromosome, their response functions characterized, and used to build the first eukaryotic UCF for S. cerevisiae BY4741 (SC1C1G1T1). Cello is then used to design large genetic circuits (up to 33,000 bp and 11 regulatory proteins) (Fig. 1). The sensors are always located at the NRT1 locus (I.ChXV) [78] and the circuits are divided between two loci (IIA.ChIII and IIB.ChV) (Supplementary Fig. 1) [79-81]. New insulators are specified to occur in a defined order, between which the transcription units corresponding to gates are positioned. A dynamic model, parameterized by the responses of individual gates, is able to predict the circuit response over weeks.

\section{Results}

\section{Genetic parts to build insulated gates}

NOT/NOR gates are based on a strong promoter that is turned off by a repressor. This promoter must not be sensitive to environmental or growth conditions. To this end, we designed synthetic constitutive promoters based on a TATA box and 20 bp TSS (transcription start site) from ADH2 (Fig. 2a) [62, 82, 83]. We varied the location and sequences of the UAS (upstream activating sequence) and region between the TATA box and TSS (Extended Data Fig. 1). From these, we selected a promoter based on Gal4-binding UAS sites flanked by two $15 \mathrm{bp}$ poly-T sequences and a $31 \mathrm{bp}$ spacer between the UAS and TATA box from the Gal1 promoter. This scaffold was used to determine how best to insert operators. Either one or two tetO operators were placed at different locations (Fig. 2b) (repeated for lacl/lacO, Extended Data Fig. 2). The promoters were fused to a $10 \mathrm{bp}$ Kozak sequence and yellow fluorescent protein gene $(\mathrm{yfp})$ and cloned into a plasmid (Supplementary Fig. 15). TetR containing a nuclear localization signal (NLS)[84] is 
constitutively expressed from a second plasmid (Supplementary Fig. 15). Fluorescence measurements were made using strains carrying the promoter reporter in presence or absence of the plasmid expressing TetR (Fig. 2b) (Methods). The strongest repression was consistently observed for promoters containing two operators separated by more than $20 \mathrm{bp}$.

To eliminate Gal4 as a regulatory input, we identified an alternative UAS that could achieve high expression levels. We found that a $20 \mathrm{bp}$ poly-T sequence that is separated from the TATA box by $4 \mathrm{bp}$ consistently led to a strong promoter that is independent of whether cells are grown in glucose or galactose (Extended Data Fig. 1). The Kozak sequence was shortened to only 10 bp (Supplementary Table 6) [85]. Collectively, these changes led to a canonical promoter structure (Psyn.0ac) that, to our knowledge, is the shortest ( $<120 \mathrm{bp}$ ) regulatable promoter for S. cerevisiae (Fig. 2a).

A repressor set was then collated, including Lacl, XyIR, LexA, a set of TetR-family repressors, and bacteriophage repressors $(\mathrm{Cl}, \mathrm{Cl} 434$, and $\mathrm{HKCl})$, from which we eliminated those that were toxic or exhibited cross-talk (not shown) [1, 86-88] [23, 25]. Based on the Psyn.0ac scaffold, we designed a set of promoters that can respond to 12 repressors (Extended Data Fig. 1, Supplementary Figs. 2 to 12). For those repressors used to build gates, two variants for each promoter were built so that they could be used without invoking homologous recombination (Fig. 2c).

Readthrough of RNAP from a gate to its neighbors can cause circuit failures [89]. The strengths of 33 yeast terminators were characterized when used to block transcription from 35 upstream promoters (Fig. 3a and Supplementary Tables 7, 8) (Methods). All 1,155 combinations were evaluated and, while there are some outliers, we find that expression level is dominated by promoter choice (Fig. 3a). When terminators appear immediately adjacent to a promoter, they can change the nucleosome occupancy and promoter activity [76]. Spacers were identified to disrupt this effect. To improve effectiveness, we placed ribozymes (RiboJ variants $[35,90,91]$ ) between the terminator and promoter. While this does not stop an errant transcript from being produced, it will be cleaved and not capped or exported from the nucleus thereby blocking expression. When a ribozyme is placed after an inducible promoter and before a reporter gene, induction no longer leads to expression (Fig. 3b and Supplementary Fig. 15). Insulators were then constructed by pairing the strongest terminators with a ribozyme and spacer and evaluated in the genome for their ability to stop an upstream inducible system (Van) to influence a neighboring inducible system (aTc) (Fig. 3c and Extended Data Fig. 3). All of the insulators are able to block interference, thus enabling the two neighboring inducible systems to function independently.

Definition of a reference promoter 
Several reference promoters have been defined for $E$. coli and used to report promoter activities in relative promoter units (RPUs) $[32,33]$. To define a similar reference promoter for S. cerevisiae, we selected PFY1 based on an analysis by Ellis and co-workers demonstrating that it is the least affected natural constitutive promoters when cells are grown in diverse conditions [11]. Like them, we inserted it into the ura3 locus, but flanked it by strong terminators and an upstream ribozyme to buffer against transcriptional readthrough from the genome (S. cerevisiae CY671int) (Fig. 4a). The fluorescence was measured by cytometry and is defined as 1 RPU (Fig. 4b and Extended Data Fig. 4).

\section{Design of sensors and gates}

A "sensor array" was constructed at the I.ChXV locus from which regulatory proteins for three small molecule sensors are expressed (Fig. 4c). The xylose sensor is based on XyIR from Bacillus licheniformis, which we identified from libraries of regulatory proteins and promoters (Supplementary Fig. 13). Three strains were constructed to measure the output promoter of each sensor integrated into IIA.ChV. The aTc, IPTG, and xylose sensors generate 1200-fold, 120-fold, and 600-fold induction, respectively (Fig. 4d, Extended Data Fig. 5). The sensors show no cross-reactivity with respect to each other's small molecules (Fig. 4e).

We constructed 9 NOT gates based on the repressors and their cognate minimal promoters. The input to the gates is the IPTG-inducible Plac promoter (Fig. 5a). The gate is encoded at IIA.ChV and the output promoter transcribing $y f p$ is integrated at IIB.ChIII. The gate is turned off by adding IPTG, but if the $x$-axis of the response function is presented in units of concentration, then it cannot be used to calculate how to connect the gate to a different input promoter. To address this, we constructed a separate strain to measure the $\mathrm{P}_{\text {lac }}$ response to IPTG and then use these data to convert the $x$-axes of the response functions to RPU (Extended Data Fig. 6), which is then fit to

$y(x)=y_{\min }+\frac{\left(y_{\max }-y_{\min }\right) K^{n}}{K^{n}+x^{n}}$

The resulting fits are shown in Fig. 5b and parameters in Supplementary Table 2 (Extended Data Fig. 7, 8). We selected Kozak sequences and output promoters to generate similar response functions. The ranges

of $y_{\min } \in[0.001,0.2]$ and $y_{\max } \in[1.5,5.1]$ span the response threshold of the gates ( 1 RPU), thus making them easier to connect. To test whether the repressors are orthogonal, we built $9 \times 9=81$ strains that varied the repressor expressed from IIA.ChV and the output promoter carried in IIB.ChIII, and no cross reactions were observed (Fig. 5c). Finally, the growth impact of each gate was measured as a function of the strength of the input promoter (Extended Data Fig. 7, 8). These data are used by Cello to predict the growth impact of the circuit and the algorithm avoids toxic combinations. $\mathrm{Cl} 434$ and QacR slow growth 
before reaching maximum repression and are, therefore, avoided by the algorithm.

The gate dynamics were quantified using a simple model that captures the characteristic times ( $\tau_{\mathrm{ON}}$ and $\left.\tau_{\mathrm{OFF}}\right)$ for a gate to switch OFF $\rightarrow \mathrm{ON}$ or ON $\rightarrow$ OFF (Methods) [28]. To perform these measurements, strains are grown under one set of conditions (with or without inducer) until reaching steady-state and then they transferred into fresh media with the opposite conditions (Methods) (Extended Data Fig. 7, 8). These data are fit to differential equation models to calculate $\tau_{\mathrm{ON}}$ and $\tau_{\mathrm{OFF}}$ (Supplementary Table 2 ). Note that the times required for inducer to be taken up by the cells and YFP to express and fold are separated from these values.

A NOR gate architecture was developed for the S. cerevisiae genome (Fig. 5d). Two copies of the repressor gene were designed with different codon usages. Each gene is paired with the same Kozak sequences and is driven by input promoter 1 or 2 . To test this NOR gate design, we used the IcaR repressor and the aTc and IPTG sensors (Fig. 5e). The gate produces the expected 2-dimensional NOR response function (Fig. 5f) that is independent of whether the copies of the repressor genes are co-located (Fig. 5g). It is simpler for Cello to compute how to connect gates if the NOR gate response function can be described as a 1-dimensional NOT gate response function, the input to which is the sum of the input promoters. We tested this for the 9 gates where the repressor genes are encoded in the IIA.ChV locus, transcribed by $P_{\text {tet }}$ and $\mathrm{P}_{\mathrm{lac}}$. Different combinations of aTc and IPTG are added and the resulting fluorescence is measured ("Measured Output", Fig. 5h). This is compared to the "Predicted Output" that is obtained when the two input promoters are summed and used with the NOT gate response function (taken from Fig. 5b). While there is some systematic error, the correlation is sufficient to use the NOT response functions to connect gates.

\section{Genetic circuit design automation}

Cello maps the desired circuit to a linear DNA sequence to be inserted in a specific genetic location. For $S$. cerevisiae, we selected two genomic loci to carry the circuit (Fig. 6a) (Supplementary Fig. 1) [81]. In practice, we found maintaining the insulator order resulted in more reliable circuits. Therefore, we constrain the order of the insulators, as opposed to an order of repressor genes/gates. This is shown in Fig. 6a, where the transcription units follow the progression of 1 to 16 , but the order of the repressor genes in the progression is random. To evaluate the difference in expression at these sites, 16 strains were built where a $\mathrm{P}_{\text {tet }}-y f p$ construct was inserted at each position and the variation in expression is < 2-fold, which is small enough to not disrupt gate function (Fig. 6a).

To validate design automation, 3-input logic circuits were constructed, each of which was initially 
specified as a Verilog truth table (Supplementary Note 1). The sensors were specified along with their OFF/ON response (red lines, Fig. 4d) and UCF SC1C1G1T1 is selected (Supplemental File). Cello then parsed the Verilog text, used logic minimization to create a wiring diagram, simulated annealing to assign repressors, and maps the result to a DNA sequence according to the constraints. The output is predicted for all combinations of inputs. The predictions were made prior to constructing the circuits and the DNA sequences were built exactly as specified. Six circuits were designed in this way, of which five functioned as predicted (Fig. 6c) (Methods). One circuit had one failed state, which we traced to the $\mathrm{Cl}$ gate population variability, which sits at the threshold of the next gate (Extended Data Fig. 9). The growth impact of the circuits was also assessed for all of their states, of which 6 out of 40 slow growth (Extended Data Fig. 10).

The dynamic responses of the five circuits were measured for $>2$ weeks (400 hours) without selective markers (Fig. 7a). The cells are exposed to different combinations of inducers every 48 hours until the circuit is transitioned through all eight states (Methods). No gross circuit failures or genetic instability was observed for any of the circuits. The circuits either have four (0x06 and 0xF6) or five layers and require much more time to reach steady-state (up to 30 hours) than the individual gates ( 12 hours). The response over time was compared to a mathematical model that is parameterized only with the empirically-measured on- and off- times of the individual gates (Supplementary Table 2) (Methods). The model accurately captures the performance of the circuit over time, including the delays due to layering.

Transiently incorrect outputs, or "faults," can occur when the signal splits and one rapidly progress to the end, whereas the other is delayed as it propagates through additional layers [92, 93]. For $S$. cerevisiae, there is a long delay for the signal to propagate through a gate and this leads to prevalent faults, which are predicted by the mathematical model. To show the relationship between the gate delays and a fault, additional experiments were performed with the 0xA1 circuit looking at the transition from $/-/-$ to $+/+/+$ (Fig. 7b). The last gate is an OR function where one input turns off faster than the other can turn on after this change in the inducers. To test this, strains were constructed that contain $r f p$ reporters for the output promoters of each intermediate gate in the circuit integrated into the HO locus (Fig. 7c, $d$ and Supplementary Table 4). Each gate is accurately modeled by the simple ODE equations, including the observed fault.

\section{Discussion}

Many groups have shown that you can combine regulatory interactions to build a circuit function that performs correctly at a gross level $[8,10,13]$. However, circuit design is performed "by hand" and the 
quantitative response is not predictable prior to experimental testing. We have found that making more precise predictions does not require a more accurate model or extensive parameterization. Instead, it requires the careful design of insulated gates and selection of where they should be carried in the cell.

Philosophically, we define the UCF for a highly-specified context (strain, loci, growth conditions) for which the predictions are valid. While the circuit may work when inserted in a different locus, strain, or media, these are not the conditions for which it was designed. Achieving precise predictions through design automation requires that the gates be re-characterized in the new strain or genetic location of interest and these data used to build a new UCF. While going through the process of building new gates and a new UCF is initially difficult, after it is complete, it greatly simplifies the design of genetic circuits for that host. In fact, a circuit function can be re-mapped to DNA for different hosts in seconds just by selecting a new UCF in Cello. We now have examples where the same circuit specified with Verilog code is specified either for an E. coli plasmid (Eco1C2G2T2) or yeast chromosome (SC1C1G1T1). The Verilog files we are currently writing are relatively simple, but one can imagine a future where genetic designs are shared at this higher and more abstract level and then re-compiled to new organisms as needed.

Large genetic engineering projects will require regulatory networks to coordinate responses; for example, turning on metabolic valves at the right times during growth, managing energy and materials resources, dividing tasks amongst a population, and staging processes. These networks must function precisely in different states and implement dynamic responses. The ability predict faults could guide circuit design for a particular application. One example would be avoiding faults for transitions where it is critical to remain in the same state; for example, if a cells is programmed to die after a fermentation, one would not want that pathway to be inadvertently induced as a fault when conditions change in the fermenter. As the need for more complex regulatory networks grows, they will become too large to construct in an ad hoc manner and have too many states to be optimized by directed evolution. Design automation will be increasingly relied on to balance the constraints and put together the multitude of DNA parts required.

\section{Acknowledgements}

This work was supported by funding from the US Department of Energy (DOE) (DE-SC0018368 to Y.C. and C.A.V), NSF SynBERC Engineering Research Center SynBERC (SA5284-11210 to C.A.V.), US Defense Advanced Research Projects Agency (DARPA) 1kM award (HR0011-12-C-0067 to C.A.V.) and SD2 (FA875017-C-0229 to S.Z. and C.A.V.), a research contract from DSM (E.M.Y.). 


\section{Author contributions}

Y.C. and C.A.V. conceived the study and designed the experiments. S.Z. performed the computational work. E.M.Y. cloned and characterized native yeast parts. Y.C. performed all other experiments and analyzed the data. Y.C., E.M.Y, and C.A.V. wrote the manuscript.

\section{Methods}

Strain, media, and reagents. Saccharomyces cerevisiae (S. cerevisiae) BY4741 MATa his3 $\Delta 1$ leu2 $\Delta 0$ met15 $\triangle 0$ ura3 $\triangle 0$, an $\mathrm{S} 288 \mathrm{C}$ derivative, was the parent strain unless otherwise noted. S. cerevisiae CENPK.113-7D was used to measure the native promoter-terminator combinations. Cells were grown in either in YPD broth (Sigma-Aldrich, Y1375) or synthetic drop-out medium (SD; Sunrise, \#1701). SD-Ura (Sunrise, \#1703), SD-Leu (Sunrise, \#1707), SD-His (Sunrise, \#1705), and SD-Ura-Leu (Sunrise, \#1721) media were used to select the correct clones for genome integration or maintain plasmids with corresponding markers. When indicated, $2 \%$ galactose (Sigma-Aldrich, G0750) was added to SD without glucose (Sunrise, \#1797) medium. The antibiotic $100 \mu \mathrm{g} / \mathrm{ml}$ nourseothricin (Gold Biotechnology, N-500) was used for NatMX marker selection. 1.8\% agar (Bacto, 214010) was used for agar plates. The inducers used are isopropyl $\beta$ D-1-thiogalactopyranoside (IPTG; Sigma-Aldrich, 16758), anhydrotetracycline hydrochloride (aTc; SigmaAldrich, 37919), D-xylose (Xyl; Sigma-Aldrich, X1500) and vanillic acid (Sigma-Aldrich, 94770). The reporter genes used for these experiments is yellow fluorescent protein (YFP) and red fluorescent protein (RFP) codon optimized for yeast [94-96].

Collation of yeast part library. Promoters and terminators were sourced from previous yeast part collections [70, 74, 97-102], yeast transcriptomic data [103], and from other yeasts in the Saccharomyces genus using the Broad Institute Fungal Orthogroups Repository [104]. Parts were mutated to eliminate Bsal and Bpil restriction enzyme sites. For parts sourced from transcriptomic data, the sequence length was adjusted to capture the region of reduced nucleosome occupancy, as defined by Segal and co-workers [105], and to avoid including proximal genome features. Promoters and terminators were synthesized by GeneArt, the DOE Joint Genome Institute, or amplified from S. cerevisiae genomic DNA or yeast shuttle vectors. The sequences of all genetic parts are provided in Supplementary Table 7 and 8.

Cell culture conditions for the characterization of parts, gates, and circuits. The following protocol was used for all promoter, terminator, and insulator experiment except those noted in the next section. Frozen stocks were streaked and grown overnight. A single colony was picked into $500 \mu \mathrm{l}$ SD medium (with 
auxotrophic selection) and inducers (if required) in $2 \mathrm{ml}$ 96-well deep well plate (Plate One, 1896-2000) sealed with AeraSeal film (Excel Scientific, BS25). The plates were incubated at $30^{\circ} \mathrm{C}$ and $900 \mathrm{rpm}$ in a Multitron shaker incubator for 24 hours. For circuits containing 2+ layers, this culture was diluted 200fold a second time and grown again under the same conditions for an additional 24 hours. Then, the overnight culture was diluted to $\mathrm{OD}_{600}=0.005$ (Synergy H1 plate reader, BioTek) by adding $2.5 \mu \mathrm{l}$ of culture to $497.5 \mu \mathrm{l}$ fresh SD medium (without selection, but with inducers if required) in $2 \mathrm{ml}$ 96-well deep well plate and sealed with AeraSeal film. The cultures are grown at $30^{\circ} \mathrm{C}$ and $900 \mathrm{rpm}$ in a Multitron shaker incubator for 16 hours. After growth, a $20 \mu \mathrm{l}$ culture was added to $180 \mu \mathrm{l}$ phosphate buffered saline (PBS; Omnipur, 6505-OP) with $10 \mathrm{\mu g} / \mathrm{ml}$ cycloheximide (Sigma-Aldrich, 227048) into a 96-well U-bottom plate (Corning, 3367) and incubated for 1 hour at room temperature for cytometry analysis. In addition, $200 \mu \mathrm{l}$ of this culture was transferred into a 96-well black walled optical bottom plates (Nunc, 165305) to measure the cell density.

Characterization of promoter-terminator pairs. The following protocol was used for promoter-terminator pairs characterization in Fig. 3a. Note that the host strain of this experiment is S. cerevisiae CENPK.113-7D, which is different from the one used for the remainder of the work in this manuscript. Expression strengths of promoter and terminator pairs was determined using Venus YFP [96] and the constructs were shown in Supplementary Fig. 16. Note that this reporter gene is different from the one used for the remainder of the work in this manuscript. A single colony of yeast transformants was picked into $500 \mu \mathrm{l}$ SD medium (with nourseothricin selection, $2 \%$ glucose or $2 \%$ galactose as carbon source) into a $2 \mathrm{ml}$ 96-well deep well plate (Plate One, 1896-2000) sealed with AeraSeal film (Excel Scientific, BS25). The plates were incubated at $30^{\circ} \mathrm{C}$ and $900 \mathrm{rpm}$ in a Multitron shaker incubator for 48 hours. Then, the culture was diluted by adding $10 \mu$ of culture into $490 \mu$ fresh SD medium (with nourseothricin selection, $2 \%$ glucose or $2 \%$ galactose as carbon source) in $2 \mathrm{ml}$ 96-well deep well plate and sealed with AeraSeal film. The cultures are grown at $30^{\circ} \mathrm{C}$ and $900 \mathrm{rpm}$ in a Multitron shaker incubator for 24 hours. After growth, $20 \mu$ of the culture was added to $180 \mu \mathrm{l}$ phosphate buffered saline (PBS; Omnipur, 6505-OP) with $10 \mu \mathrm{g} / \mathrm{ml}$ cycloheximide (SigmaAldrich, 227048) into a 96-well U-bottom plate (Corning, 3367) and incubated for 1 hour at room temperature for cytometry analysis.

Flow cytometry. The fluorescence was measured for $>20000$ events of each sample with LSRII Fortessa flow cytometer with HTS (BD Biosciences). FITC-A and PE Texas-A channels were chosen for YFP and RFP measurement respectively. Data was processed by FlowJo (TreeStar) to obtain the median of 
fluorescence, gated by the width of the forward scatter and the side scatter (FSC-W/SSC-W)[23]. The Foldrepression was calculated by (YFPunrepressed-YFPo)/ (YFPrepressed-YFPo); The fold-induction was calculated by $\left(\mathrm{YFP}_{\text {induced }}-\mathrm{YFP}\right.$ )/ (YFP uninduced-YFPo). YFP unrepressed $_{\text {and }}$ YFP repressed are the fluorescence of the ON and OFF states of NOT gate strain (YFP as reporter); YFP induced and YFP uninduced are the fluorescence of the sensor strain with (100ng/ml aTc, $10 \mathrm{mM}$ xylose, and $20 \mathrm{mM}$ IPTG respectively) and without inducer; $\mathrm{YFP}_{0}$ is that from white cells (S. cerevisiae BY4741).

Calculation of RPU. To characterize a promoter, the median fluorescence values (YFP) were measured along with the fluorescence of the RPU reference strain (S. cerevisiae CY671int) (YFPRP) and that from white cells (S. cerevisiae BY4741) (YFP0), all under the same growth conditions as described above. The promoter activity in RPU was calculated by RPU $=\left(\mathrm{YFP}_{\mathrm{Y}} \mathrm{YFP}\right) /\left(\mathrm{YFP}_{\mathrm{RPU}}-\mathrm{YFP}_{0}\right)$.

Measurement of growth rates. Cell density was measured as $\mathrm{OD}_{600}$ with a Synergy $\mathrm{H} 1$ plate reader (BioTek). We also measured background $\mathrm{OD}_{600}$ using a $200 \mu \mathrm{l}$ aliquot of media without cells, which was subtracted from the $\mathrm{OD}_{600}$ value for a sample. When presented as "normalized," the measured value is divided by that measured for the same strain without inducers.

Measurement of gate and sensor dynamics. Colonies are picked into $500 \mu \mathrm{l}$ SD medium (with auxotrophic selection) and inducers (initial states, if required) in $2 \mathrm{ml}$ 96-well deep well plate (Plate One, 1896-2000) sealed with AeraSeal film (Excel Scientific, BS25). The plates were incubated at $30^{\circ} \mathrm{C}$ and 900 rpm in a Multitron shaker incubator for 24 hours. Then, the overnight culture was diluted to $O D_{600}=0.005$ (Synergy H1 plate reader, BioTek) by adding $2.5 \mu$ of culture to $497.5 \mu$ fresh SD medium (without selection, but with initial state inducers if required) in $2 \mathrm{ml}$ 96-well deep well plate and sealed with AeraSeal film. The cultures were grown at $30^{\circ} \mathrm{C}$ and $900 \mathrm{rpm}$ in a Multitron shaker incubator for 16 hours. Then, $500 \mu \mathrm{l}$ of the culture collected at $\mathrm{OD}_{600}=1.0$ was spun down by centrifugation at $4^{\circ} \mathrm{C}$ at $500 \mathrm{~g}$ for 5 min. After removing the supernatants, the cell pellet was resuspended in $500 \mu$ fresh SD-glucose media (with final state inducers) and then 1:20 diluted to $500 \mu$ l fresh SD-glucose media (with final state inducers) at $\mathrm{OD}_{600}=0.05$ (Synergy $\mathrm{H} 1$ plate reader, BioTek) in $2 \mathrm{ml}$ 96- deep well plate sealed with AeraSeal film. The plates were incubated at $30^{\circ} \mathrm{C}$ and $900 \mathrm{rpm}$ in a Multitron shaker incubator. Aliquots $(20 \mu \mathrm{l})$ of the culture were collected every 3 hours for flow cytometry measurement. During the measurement, the culture was 1:10 diluted to $\mathrm{OD}_{600}=0.05$ in $500 \mu$ l fresh SD-glucose media (with final state inducers) for every 12 hours. 
Model of gate dynamics. The model follows the approach described previously and the detailed derivation and fitting procedure are presented in that manuscript [28]. Briefly, the model has two parameters that capture how quickly a gate moves to its steady-state output given the state of the input. For example, if the input is ON and the gate output $y$ is OFF, then if the input changes to being OFF, the gate output is lower than its steady-state value $y_{s s}$ and it will turn on at a rate dictated by the parameter TON. In the opposite case, when the input is OFF and then turns ON, then $y$ will be higher than $y_{s s}$ and have to turn off, which it will do at a rate following the parameter $\tau_{\text {OFF. }}$ This response is captured by the following differential equation describing a single gate:

$\frac{d y}{d t}=\left\{\begin{array}{c}\tau_{y}^{O N}\left(y_{s s}-y\right) \text { if } y<y_{s s} \\ \tau_{y}^{O F F}\left(y_{s s}-y\right) \text { otherwise }\end{array}\right.$

where the relationship between $y_{s s}$ and the input $x$ is provided by Equation 1 . Note that $d y / d t$ describes the rate of change of the RNAP flux from the promoter and not the reporter expression and maturation, which is separated. We developed a fitting procedure to extract the parameters $\tau_{\text {ON }}$ and $\tau_{\text {OFF }}$ using the empirical measurements described in the previous section. First, the dynamic response of the sensor is measured (Extended Data Fig. 5), from which we extract the response time of the sensor's output promoter upon addition or removal of the inducer and the rate associated with the expression and folding of the reporter protein. YFP degradation is assumed to be dominated by the cell doubling time. To fit the data for each sensor, the following equations are used

$\frac{d x}{d t}=\tau_{x}\left(x_{s S}-x\right)$ and

$\frac{d[Y F P]}{d t}=\tau_{O N}^{Y F P} x-\tau_{O F F}^{Y F P}[Y F P]$,

where $x$ is the RNAP flux from sensor's output promoter reported in RPU. The results from this process are $\tau_{\text {ON }} / \tau_{\text {OFF }}$ of the IPTG, aTc, and Xyl sensor of $0.2 / 0.25,0.3 / 0.3$, and $0.3 / 0.3$. The YFP response is $\tau_{\text {ON }}{ }^{\text {YFP }}$ $=944 \mathrm{au}-\mathrm{RPU}^{-1} \mathrm{hr}^{-1}$ and $\tau_{\mathrm{OFF}}^{\mathrm{YFP}}=2.3 \mathrm{hr}^{-1}$. Then, the on and off dynamics of each gate was measured. To fit the data for each gate, the following equations are used

$\frac{d x}{d t}=\tau_{x}\left(x_{s s}-x\right)$,

$\frac{d y}{d t}=\tau_{y}\left(y_{s s}-y\right)$ and

$\frac{d[Y F P]}{d t}=\tau_{O N}^{Y F P} y-\tau_{O F F}^{Y F P}[Y F P]$,

The results of the fitting procedure are shown in Extended Data Fig. 7, 8 and the parameters for each gate are in Supplementary Table 2.

User Constraint File (UCF). The S. cerevisiae UCF (SC1C1G1T1) was created is provided in the 
Supplementary Data. The UCF contains the gate technology and associated data (response functions, including cytometry distributions, and the $\mathrm{OD}_{600}$ measurements). It also defines the strain, genetic location of the circuit, and the growth conditions where the circuit design is valid. SC1C1G1T1 is based on S. cerevisiae strain BY4741 and the location of the circuits is ura3:: (IIA.ChV) and leu2:: (IIB.ChIII) (Fig. 1). For each gate, the repressor has two versions of sequences in different codon to minimize potential homolog sequence; and the targeting promoter also have a mutated version for the same reason. Eugene [106] rules are also included to specify the organization of gates onto a linear DNA sequence. This UCF uses the following layout rules for gates and Type IIS cloning scars. The use of multiple gates that use the same repressor and different targeting promoters are prohibited from appearing in the same circuit. Cloning scars appear in the order $\mathrm{A}-\mathrm{B}-\mathrm{C}-\mathrm{D}-\mathrm{E}-\mathrm{F}-\mathrm{G}-\mathrm{H}-\mathrm{I}$ (IIA.ChV) and $\mathrm{J}-\mathrm{K}-\mathrm{L}-\mathrm{M}-\mathrm{N}-\mathrm{P}-\mathrm{Q}-\mathrm{R}$ -S (IIB.ChIII) (Supplementary Figs. 14, 17).

Computational circuit design. The Cello 2.0 software was used to design the circuit DNA sequences (note that the UCF will not work with the first version of Cello). The toxicity cut-off is 0.7 for gates and 0.5 for the complete circuit. Each circuit was specified as a Verilog text file (Supplementary Note 1). All circuits were specified as truth tables and Cello identified the wiring diagram using logic minimization. The SC1C1G1T1 UCF was used for the calculations and is provided as Supplementary Data. Data for the sensors were also provided (Supplementary Table 1). The output of Cello includes DNA sequences for the circuit, which were constructed as specified, and predictions for cytometry fluorescence data and the impact on growth.

Circuit long-term growth and induction experiments. Colonies are picked into $500 \mu \mathrm{L}$ SD medium (with auxotrophic selection) and inducers (initial states, if required) in $2 \mathrm{ml}$ 96-well deep well plate (Plate One, 1896-2000) sealed with AeraSeal film (Excel Scientific, BS25). The plates were incubated at $30^{\circ} \mathrm{C}$ and 900 rpm in a Multitron shaker incubator for 24 hours. For circuits containing 2+ layers, this culture was diluted 200-fold a second time and grown again under the same conditions for an additional 24 hours. Then, the overnight culture was diluted to $\mathrm{OD}_{600}=0.005$ (Synergy $\mathrm{H} 1$ plate reader, BioTek) by adding $2.5 \mu$ l of culture into $497.5 \mu \mathrm{l}$ fresh SD medium (without selection, but with initial state inducers if required) in $2 \mathrm{ml} 96$ well deep well plate and sealed with AeraSeal film. The cultures are grown at $30^{\circ} \mathrm{C}$ and $900 \mathrm{rpm}$ in a Multitron shaker incubator for 16 hours. Aliquots of $500 \mu \mathrm{l}$ of the culture at $O D_{600}=1.0$ were taken and spun down by centrifugation at $4^{\circ} \mathrm{C}$ at $500 \mathrm{~g}$ for $5 \mathrm{~min}$. After removing the supernatants, the cell pellet was resuspended in $500 \mu \mathrm{l}$ fresh SD-glucose media (with final state inducers) and then 1:20 diluted to 500 
$\mu$ fresh SD-glucose media (with final state inducers) at $\mathrm{OD}_{600}=0.05$ (Synergy $\mathrm{H} 1$ plate reader, BioTek) in $2 \mathrm{ml}$ 96- deep well plate sealed with AeraSeal film. The plates were incubated at $30^{\circ} \mathrm{C}$ and $900 \mathrm{rpm}$ in a Multitron shaker incubator. The same dilution process (without spin down step) was repeated every 12 hours for continuous exponential growth. The cells were collected every 3 hours for flow cytometer measurement. For the dynamic experiments performed over 400 hours, cells were resuspended and diluted to $\mathrm{OD}_{600}=0.005$ (Synergy $\mathrm{H} 1$ plate reader, BioTek) in fresh SD glucose media with inducers every 24 hours. The fluorescence was measured by flow cytometry at 16 hours after dilution.

Simulation of circuit dynamics. The RPU outputs for all intermediate gates at initial condition were first obtained from Cello. Then, performed a numerical analysis from time 0 using the ODEs for the circuit. For example, the equations for the $0 x 06$ circuit are shown below:

$\frac{d t e t R}{d t}=\tau_{\text {tetR }}\left(\right.$ tet $R_{\text {sS }}-$ tet $\left.R\right)$,

$\frac{d x y l R}{d t}=\tau_{x y l R}\left(x y l R_{S S}-x y l R\right)$,

$\frac{d l a c I}{d t}=\tau_{l a c I}\left(l_{a c I_{s S}}-l a c I\right)$,

$\frac{d i c a R}{d t}=\tau_{i c a R}\left(i c a R_{S S}-i c a R\right)$,

$\frac{d \operatorname{lexA}}{d t}=\tau_{\operatorname{lexA}}\left(\operatorname{lex} A_{S S}-\operatorname{lex} A\right)$,

$\frac{d p h l F}{d t}=\tau_{p h l F}\left(p h l F_{S S}-p h l F\right)$,

$\frac{d c I}{d t}=\tau_{c I}\left(c I_{s S}-c I\right)$,

$\frac{d c I 434}{d t}=\tau_{c I 434}\left(c I 434_{s S}-c I 434\right)$,

$\frac{d b m 3 R 1}{d t}=\tau_{b m 3 R 1}\left(b m 3 R 1_{s s}-b m 3 R 1\right)$,

$\frac{d[Y F P]}{d t}=\tau_{O N}^{Y F P} b m 3 R 1-\tau_{O F F}^{Y F P}[Y F P]$,

Where tetR, xylR and lacl are the sensor inputs and icaR, lexA, phIF, $\mathrm{cl}, \mathrm{cl} 434$ and bm3R1 are the gates outputs. The equations were solved by Matlab scripts, using the ordinary differential equation solver ODE15s. All scripts are available on Github (https://github.com/VoigtLab). 


\section{Figure Captions}

Fig. 1: S. cerevisiae circuit design using Cello. The sensors (A, B, C), Verliog code for the circuit, and UCF are defined. Cello parses the Verilog text, uses logic minimization to create a wiring diagram, and simulated annealing to assign repressors to each gate (colors). Finally, the composition rules are used to map the circuit to a DNA sequence. The position of the chromosomes is approximated using data from reference [81].

Fig. 2: Design of minimal synthetic promoters that respond to regulators. (a) The canonical promoter design is shown. The TATA box (blue) is TATAAAAA. Sequences consisting of 20 T nucleotides are place upstream of the TATA box and between the operators; mutations are made to these sequences to avoid recombination. The TSS region is sourced from $20 \mathrm{bp}$ from the natural ADH promoter and the Kozak sequence is a synthetic 10bp sequence [85]. All genetic parts are provided in Supplementary Table 5, S7 and S8. (b) Comparison of operator spacing. The promoter backbone is Psyn.0 with a Gal4 UAS (Supplementary Fig. 2) and is cloned into a reporter plasmid (Supplementary Fig. 15). A second plasmid is constructed where TetR is expressed from a constitutive promoter (Supplementary Fig. 15). The cytometry distributions show the fluorescence from the reporter plasmid in the absence (black) and presence (grey) of the TetR plasmid and the fold change is the ratio of the median of these distributions. (c) The architectures of the final synthetic promoters and their strengths are shown. The colors correspond to part a, the sequences are provided in Supplementary Table 5, and the promoters were integrated into the IIA.ChV (sensor promoters) or IIB.ChIII (gate promoters) genomic loci. The step-bystep design of each promoter is described in Extended Data Fig. 1 and Supplementary Figs. 2 to 12. The mutations made to diversify the stretches of T nucleotides are shown as vertical lines; two promoters were selected for promoters used for gates (and one for those used in sensors). The lower dashed line marks the fluorescence of white cells and the upper dashed line is the strong $\mathrm{P}_{\mathrm{TDH}}$ promoter. In all parts, the data represent the average of three experiments performed on different days, the standard deviation of which is shown as the error bars.

Fig. 3: S. cerevisiae terminator and insulators. (a) All combinations of promoters and terminators were tested for their ability to express a YFP reporter when grown in media containing glucose (Methods and Supplementary Fig. 16. Note that these measurements were made in a different strain background ( $S$. cerevisiae CENPK.113-7D) than the remainder of the experiments in this manuscript. The grid represents single data points taken during the screen. The DNA sequences of the promoters and terminators are 
provided in Supplementary Table 7 and 8, respectively. (b) Evaluation of ribozyme activity in blocking mRNA export. The ribozyme is placed after an aTc-inducible promoter and before the $y f p$ reporter on a plasmid (Supplementary Fig. 15). The fold-change of the fluorescence is shown as the ratio in the presence and absence of $100 \mathrm{ng} / \mathrm{ml}$ aTc. These data represent the average of three experiments performed on different days. (c) Measurement of readthrough between two adjacent transcription units. Different terminator/ribozyme/spacer combinations were tested using the system shown (the grey gene is $r f p$, but this is only used as a spacer). The bars show the ratio of $y f p$ fluorescence measured upon the induction of the upstream sensor (+/- $200 \mu \mathrm{M}$ vanillic acid). Two transcription units were also integrated into different chromosomes (IIA.ChV and IIB.ChIII) for comparison ("Separated loci"). Two 450bp non-functional DNA spacer sequences (S1 and S2) were chosen as a control for the absence of an insulator. In all parts, the data represent the average of three experiments performed on different days, the standard deviation of which is shown as the error bars.

Fig. 4: Small molecule sensors. (a) The RPU reference promoter is shown. The DNA sequence is provided in Supplementary Table 11. (b) The arbitrary units of fluorescence are converted to RPU by dividing by the strength of this promoter. The upper dashed line represents the strong $\mathrm{P}_{\mathrm{TDH} 3}$ promoter and the lower dashed line is the background fluorescence of white cells. The cytometry histograms associated with these measurements are shown in Extended Data Fig. 4. The data represent the average of three experiments performed on different days, the standard deviation of which is shown as the error bar. (c) The three sensors were inserted into the genome. A representative reporter construct for the aTc-inducible system is shown (right). The repressors for each inducible system were placed under the control of a constitutive promoter ( $\mathrm{P}_{\mathrm{VMAG}}, \mathrm{P}_{\mathrm{FBA} 1}$, and $\mathrm{P}_{\mathrm{PXR} 1}$ ). A natMX gene was also added for colony selection. The sequences for all of the genetic parts are provided in Supplementary Table 5, 8. (d) The response functions of the three sensors are shown. Each was measured with a reporter construct integrated at the IIA.ChV locus in the genome (Supplementary Fig. 14). Only the OFF/ON values of each sensor are used as inputs to Cello; OFF is no inducer and the $\mathrm{ON}$ values are shown as dashed red lines $(100 \mathrm{ng} / \mathrm{ml}$ aTc, $10 \mathrm{mM}$ D-xylose, and 20 mM IPTG). The curves represent the average of three experiments performed on different days. (e) No crosstalk is observed between the three sensors. The concentrations of small molecules are: $100 \mathrm{ng} / \mathrm{ml}$ aTc, $10 \mathrm{mM}$ D-xylose, and $20 \mathrm{mM}$ IPTG. The data represent the average of three experiments performed on different days. Fold-induction is calculated as RPU (+ inducer)/ RPU (-inducer).

Fig. 5: NOT/NOR gates. (a) The NOT gate design is based on repressors that contain a C-terminal NLS (black). (b) The IPTG inducible system ( $\mathrm{P}_{\text {lac }}$ is used to measure the response of each gate. Using a separate 
strain, the strength of $\mathrm{P}_{\mathrm{lac}}$ is measured independently as a function of inducer concentration (Extended Data Fig. 6a). These data are used to change the $x$-axis of the response function of the NOT gate from units of inducer concentration to the activity of the input promoter in RPU. The data for the response of each gate is fit to a function (color coded by repressor). The data used for these fits are shown in Extended Data Fig. 7, 8. The concentrations of IPTG (from left to right) are: 0, 0.1, 0.25, 0.5, 1, 1.5, 2, 2.5, 3, 5, 10 and 20. (c) The orthogonality of the gates. The promoters and reporters are integrated into IIB.ChIII and the repressors ( $P_{\text {tet }}$ controlled) are integrated into IIA.ChV under aTc control ( 0 or $100 \mathrm{ng} / \mu \mathrm{l}$ aTc added). The fold-repression is calculated as RPU (-aTc)/ RPU (+aTc). (d) The design of the NOR gate is shown, where each input promoter transcribes a different copy of the repressor gene with different codon assignments. (e) The two copies of the repressor are integrated at the same genomic locus (top) or at two loci (bottom). (f) The response of the IcaR NOR gate at IIA.ChV is shown as a function of both inducers. IPTG is added at $0,0.5,2$, and $20 \mathrm{mM}$ (Input 1) and aTc is added at 0, 20, 30, and $100 \mathrm{ng} / \mathrm{ml}$ (Input 2). (g) The IcaR NOR gate is compared when the repressor genes are on the same chromosome or split between two (as shown in part e). (h) Comparison of the output of a NOR gate to that which is predicted by using the corresponding NOT gate response function. For all 9 gates, the 2-D response is measured (as in part $\mathbf{f}$ ) and compared to that predicted using the 1-D response function (part $\mathbf{b}$ ) and summing the activities of the input promoters. The data represent the average of three experiments (part $\mathbf{c}, \mathbf{f}$ ) or the individual experiments (part $\mathbf{g}, \mathbf{h}$ ) performed on different days.

Fig. 6: Genetic circuits. (a) Each circuit occupies two chromosomal locations. The insulators are in a defined order and the gates are assigned to positions 1 to 16 until there are no more gates associated with the circuit. The remaining insulators that are not used are not included in the circuit DNA. (b) The expression differences when an aTc-inducible promoter and $y f p$ reporter are inserted at each position. For these measurements, the insulators before and after the position are included unless it appears at an edge. Relative strength is the measured fluorescence (with $100 \mathrm{ng} / \mu \mathrm{laTc}$ ), reported relative to position 1. The data represent the average of three experiments performed on different days, the standard deviation of which is shown as the error bars. (c) Circuits designed by Cello. The boxes correspond to the positions in part $\mathbf{a}$ and the squares represent the insulators. Cytometry distributions are shown for the circuit output in the presence or absence of $100 \mathrm{ng} / \mathrm{ml} \mathrm{aTc}, 10 \mathrm{mM}$ xylose and $20 \mathrm{mM} \mathrm{IPTG}$ (Methods). The solid red and blue distributions are those predicted by Cello when the output is low or high, respectively. For all histograms, at least three experiments were performed on different days with similar results. 
Fig. 7: Circuit dynamics. (a) The five circuits were cycled between states for 400 hours (17 days).

Cells containing the circuits were cultured as described in the Methods, diluting into fresh media daily. Every two days, different combinations of inducers were added to the media (shown at the top). The experimental data points are shown and compared to the dynamics predicted with the ODE (Methods). The data points represent biological triplicates, collected concurrently. (b) The circuit diagram of 0xA1 is shown including the promoters corresponding to each "wire." (c) Cells containing the circuit were grown with the -/-/- combination of inducers until steady-state and then transferred into fresh media with the $+/+/+$ combination of inducers. The response of each of the sensors are gates to this transition are shown. The sensors are measured using strains that only contain their output promoter fused to $y f p$ (no circuit) (S. cerevisiae CY637int, CY639int, CY928int) (Supplementary Table 4). The other wires were analyzed by fusing the promoter shown in part $\mathbf{b}$ to red fluorescent protein $(r f p)$ and integrating the reporter into the Chromosome IV , 48031...46271, HO locus. The points are experimental duplicates collected over different days. The lines are the predictions from the ODE model. (d) The output of the circuit (X) is shown for the -/-/- to +/+/+ transition, highlighting a fault. The circuit diagrams show the wires with active RNAP flux at the different time points. The middle diagram shows the delay leading to the fault when one of the inputs to the last NOR gate is turned off before the other can turn on. The data represent two experiments performed on different days.

\section{References}

1. Ellis, T., X. Wang, and J.J. Collins, Diversity-based, model-guided construction of synthetic gene networks with predicted functions. Nat Biotechnol, 2009. 27(5): p. 465-71.

2. $\quad$ Krivoruchko, A., V. Siewers, and J. Nielsen, Opportunities for yeast metabolic engineering: Lessons from synthetic biology. Biotechnol J, 2011. 6(3): p. 262-76.

3. Billingsley, J.M., A.B. DeNicola, and Y. Tang, Technology development for natural product biosynthesis in Saccharomyces cerevisiae. Curr Opin Biotechnol, 2016. 42: p. 74-83.

4. Geva, P., et al., Increased copper bioremediation ability of new transgenic and adapted Saccharomyces cerevisiae strains. Environ Sci Pollut Res Int, 2016. 23(19): p. 19613-25. 
5. Qian, S. and P.C. Cirino, Using metabolite-responsive gene regulators to improve microbial biosynthesis. Curr Opin Chem Engin, 2016. 14: p. 93-102.

6. Ferreira, R., et al., Model-Assisted Fine-Tuning of Central Carbon Metabolism in Yeast through dCas9-Based Regulation. ACS Synth Biol, 2019. 8(11): p. 2457-2463.

7. Ajo-Franklin, C.M., et al., Rational design of memory in eukaryotic cells. Genes Dev, 2007. 21(18): p. 2271-6.

8. Marucci, L., et al., How to turn a genetic circuit into a synthetic tunable oscillator, or a bistable switch. PloS one, 2009. 4(12): p. e8083.

9. Buchler, N.E. and F.R. Cross, Protein sequestration generates a flexible ultrasensitive response in a genetic network. Mol Syst Biol, 2009. 5: p. 272.

10. Regot, S., et al., Distributed biological computation with multicellular engineered networks. Nature, 2011. 469(7329): p. 207-11.

11. Blount, B.A., T. Weenink, and T. Ellis, Construction of synthetic regulatory networks in yeast. FEBS Lett, 2012. 586(15): p. 2112-21.

12. Yamanishi, M. and T. Matsuyama, A modified Cre-lox genetic switch to dynamically control metabolic flow in Saccharomyces cerevisiae. ACS Synth Biol, 2012. 1(5): p. 172 80.

13. Youk, H. and W.A. Lim, Secreting and sensing the same molecule allows cells to achieve versatile social behaviors. Science, 2014. 343(6171): p. 1242782.

14. Ryu, J. and S.-H. Park, Simple synthetic protein scaffolds can create adjustable artificial MAPK circuits in yeast and mammalian cells. Sci. Signal., 2015. 8(383): p. ra66-ra66.

15. Khakhar, A., et al., Cell-cell communication in yeast using auxin biosynthesis and auxin responsive CRISPR transcription factors. ACS synthetic biology, 2015. 5(4): p. 279-286.

16. Rantasalo, A., et al., Synthetic Transcription Amplifier System for Orthogonal Control of Gene Expression in Saccharomyces cerevisiae. PLoS One, 2016. 11(2): p. e0148320.

17. Aranda-Diaz, A., et al., Robust Synthetic Circuits for Two-Dimensional Control of Gene Expression in Yeast. ACS Synth Biol, 2017. 6(3): p. 545-554.

18. Ryo, S., et al., Positive Feedback Genetic Circuit Incorporating a Constitutively Active Mutant Gal3 into Yeast GAL Induction System. ACS Synth Biol, 2017. 6(6): p. 928-935.

19. Chen, B., et al., Synthetic biology toolkits and applications in Saccharomyces cerevisiae. Biotechnol Adv, 2018. 36(7): p. 1870-1881.

20. Yang, Y., J.L. Nemhauser, and E. Klavins, Synthetic Bistability and Differentiation in Yeast. ACS Synth Biol, 2019. 8(5): p. 929-936.

21. Ng, A.H., et al., Modular and tunable biological feedback control using a de novo protein switch. Nature, 2019. 572(7768): p. 265-269.

22. Langan, R.A., et al., De novo design of bioactive protein switches. Nature, 2019. 572(7768): p. 205-210.

23. Ottoz, D.S., F. Rudolf, and J. Stelling, Inducible, tightly regulated and growth conditionindependent transcription factor in Saccharomyces cerevisiae. Nucleic Acids Res, 2014. 42(17): p. e130.

24. Gander, M.W., et al., Digital logic circuits in yeast with CRISPR-dCas9 NOR gates. Nat Commun, 2017. 8: p. 15459.

25. Zong, Y., et al., Insulated transcriptional elements enable precise design of genetic circuits. Nat Commun, 2017. 8(1): p. 52.

26. Bashor, C.J., et al., Complex signal processing in synthetic gene circuits using cooperative regulatory assemblies. Science, 2019. 364(6440): p. 593-597. 
27. Nielsen, A.A., et al., Genetic circuit design automation. Science, 2016. 352(6281): p. aac7341.

28. Shin, J., et al., Programming Escherichia coli to function as a digital display. Mol Syst Biol, 2020. 16(3): p. e9401.

29. Taketani, M., et al., Genetic circuit design automation for the gut resident species Bacteroides thetaiotaomicron. Nat Biotechnol, 2020.

30. Tamsir, A., J.J. Tabor, and C.A. Voigt, Robust multicellular computing using genetically encoded NOR gates and chemical 'wires'. Nature, 2011. 469(7329): p. 212-5.

31. Nielsen, A.A. and C.A. Voigt, Multi-input CRISPR/Cas genetic circuits that interface host regulatory networks. Mol Syst Biol, 2014. 10: p. 763.

32. Canton, B., A. Labno, and D. Endy, Refinement and standardization of synthetic biological parts and devices. Nat Biotechnol, 2008. 26(7): p. 787-93.

33. Kelly, J.R., et al., Measuring the activity of BioBrick promoters using an in vivo reference standard. J Biol Eng, 2009. 3: p. 4.

34. Davis, J.H., A.J. Rubin, and R.T. Sauer, Design, construction and characterization of a set of insulated bacterial promoters. Nucleic Acids Res, 2011. 39(3): p. 1131-41.

35. Lou, C., et al., Ribozyme-based insulator parts buffer synthetic circuits from genetic context. Nat Biotechnol, 2012. 30(11): p. 1137-42.

36. Chen, Y.J., et al., Characterization of 582 natural and synthetic terminators and quantification of their design constraints. Nat Methods, 2013. 10(7): p. 659-64.

37. Nielsen, A.A., T.H. Segall-Shapiro, and C.A. Voigt, Advances in genetic circuit design: novel biochemistries, deep part mining, and precision gene expression. Curr Opin Chem Biol, 2013. 17(6): p. 878-92.

38. Carr, S.B., J. Beal, and D.M. Densmore, Reducing DNA context dependence in bacterial promoters. PLoS One, 2017. 12(4): p. e0176013.

39. Brown, C.R. and P.A. Silver, Transcriptional regulation at the nuclear pore complex. Curr Opin Genet Dev, 2007. 17(2): p. 100-6.

40. Deniz, O., et al., Physical properties of naked DNA influence nucleosome positioning and correlate with transcription start and termination sites in yeast. BMC Genomics, 2011. 12: p. 489.

41. Jansen, A., et al., Distal chromatin structure influences local nucleosome positions and gene expression. Nucleic Acids Res, 2012. 40(9): p. 3870-85.

42. Curran, K.A., et al., Design of synthetic yeast promoters via tuning of nucleosome architecture. Nat Commun, 2014. 5: p. 4002.

43. Ptak, C., J.D. Aitchison, and R.W. Wozniak, The multifunctional nuclear pore complex: a platform for controlling gene expression. Curr Opin Cell Biol, 2014. 28: p. 46-53.

44. Nguyen, H.Q. and G. Bosco, Gene Positioning Effects on Expression in Eukaryotes. Annu Rev Genet, 2015. 49: p. 627-46.

45. Porrua, O. and D. Libri, Transcription termination and the control of the transcriptome: why, where and how to stop. Nat Rev Mol Cell Biol, 2015. 16(3): p. 190-202.

46. Uwimana, N., et al., Bidirectional terminators in Saccharomyces cerevisiae prevent cryptic transcription from invading neighboring genes. Nucleic Acids Res, 2017. 45(11): p. 6417-6426.

47. Riethoven, J.J., Regulatory regions in DNA: promoters, enhancers, silencers, and insulators. Methods Mol Biol, 2010. 674: p. 33-42. 
48. Gaszner, M. and G. Felsenfeld, Insulators: exploiting transcriptional and epigenetic mechanisms. Nat Rev Genet, 2006. 7(9): p. 703-13.

49. Scott, K.C., S.L. Merrett, and H.F. Willard, A heterochromatin barrier partitions the fission yeast centromere into discrete chromatin domains. Curr Biol, 2006. 16(2): p. 11929.

50. West, A.G., M. Gaszner, and G. Felsenfeld, Insulators: many functions, many mechanisms. Genes Dev, 2002. 16(3): p. 271-88.

51. Juven-Gershon, T. and J.T. Kadonaga, Regulation of gene expression via the core promoter and the basal transcriptional machinery. Dev Biol, 2010. 339(2): p. 225-9.

52. Hubmann, G., J.M. Thevelein, and E. Nevoigt, Natural and modified promoters for tailored metabolic engineering of the yeast Saccharomyces cerevisiae. Methods Mol Biol, 2014. 1152: p. 17-42.

53. Sun, J., et al., Cloning and characterization of a panel of constitutive promoters for applications in pathway engineering in Saccharomyces cerevisiae. Biotechnol Bioeng, 2012. 109(8): p. 2082-92.

54. Tirosh, I. and N. Barkai, Two strategies for gene regulation by promoter nucleosomes. Genome Res, 2008. 18(7): p. 1084-91.

55. Rando, O.J. and F. Winston, Chromatin and transcription in yeast. Genetics, 2012. 190(2): p. 351-87.

56. Redden, H. and H.S. Alper, The development and characterization of synthetic minimal yeast promoters. Nat Commun, 2015. 6: p. 7810.

57. Curran, K.A., et al., Short Synthetic Terminators for Improved Heterologous Gene Expression in Yeast. ACS Synth Biol, 2015. 4(7): p. 824-32.

58. Blazeck, J., et al., Controlling promoter strength and regulation in Saccharomyces cerevisiae using synthetic hybrid promoters. Biotechnol Bioeng, 2012. 109(11): p. 288495.

59. Lee, T.J., et al., Suppression of expression between adjacent genes within heterologous modules in yeast. G3 (Bethesda), 2014. 4(1): p. 109-16.

60. Weiner, A., et al., High-resolution nucleosome mapping reveals transcription-dependent promoter packaging. Genome Res, 2010. 20(1): p. 90-100.

61. Hodges, C., et al., Nucleosomal fluctuations govern the transcription dynamics of RNA polymerase II. Science, 2009. 325(5940): p. 626-8.

62. Lubliner, S., L. Keren, and E. Segal, Sequence features of yeast and human core promoters that are predictive of maximal promoter activity. Nucleic Acids Res, 2013. 41(11): p. 5569-81.

63. Raveh-Sadka, T., M. Levo, and E. Segal, Incorporating nucleosomes into thermodynamic models of transcription regulation. Genome Res, 2009. 19(8): p. 1480-96.

64. Raveh-Sadka, T., et al., Manipulating nucleosome disfavoring sequences allows fine-tune regulation of gene expression in yeast. Nat Genet, 2012. 44(7): p. 743-50.

65. Sharon, E., et al., Probing the effect of promoters on noise in gene expression using thousands of designed sequences. Genome Res, 2014. 24(10): p. 1698-706.

66. Sharon, E., et al., Inferring gene regulatory logic from high-throughput measurements of thousands of systematically designed promoters. Nat Biotechnol, 2012. 30(6): p. 521-30.

67. Lykke-Andersen, S., C.K. Mapendano, and T.H. Jensen, An ending is a new beginning: transcription termination supports re-initiation. Cell Cycle, 2011. 10(6): p. 863-5. 
68. Shandilya, J. and S.G. Roberts, The transcription cycle in eukaryotes: from productive initiation to RNA polymerase II recycling. Biochim Biophys Acta, 2012. 1819(5): p. 391400.

69. Grzechnik, P., S.M. Tan-Wong, and N.J. Proudfoot, Terminate and make a loop: regulation of transcriptional directionality. Trends Biochem Sci, 2014. 39(7): p. 319-27.

70. Curran, K.A., et al., Use of expression-enhancing terminators in Saccharomyces cerevisiae to increase $m R N A$ half-life and improve gene expression control for metabolic engineering applications. Metab Eng, 2013. 19: p. 88-97.

71. Ito, Y., et al., Characterization of five terminator regions that increase the protein yield of a transgene in Saccharomyces cerevisiae. J Biotechnol, 2013. 168(4): p. 486-92.

72. Morse, N.J., et al., Yeast Terminator Function Can Be Modulated and Designed on the Basis of Predictions of Nucleosome Occupancy. ACS Synth Biol, 2017. 6(11): p. 20862095.

73. Wei, L., et al., Characterization of Terminators in Saccharomyces cerevisiae and an Exploration of Factors Affecting Their Strength. Chembiochem, 2017. 18(24): p. 24222427.

74. Yamanishi, M., et al., A genome-wide activity assessment of terminator regions in Saccharomyces cerevisiae provides a "terminatome" toolbox. ACS Synth Biol, 2013. 2(6): p. 337-47.

75. Yamanishi, M., S. Katahira, and T. Matsuyama, TPS1 terminator increases $m R N A$ and protein yield in a Saccharomyces cerevisiae expression system. Biosci Biotechnol Biochem, 2011. 75(11): p. 2234-6.

76. Song, W., et al., Can terminators be used as insulators into yeast synthetic gene circuits? J Biol Eng, 2016. 10: p. 19.

77. Yarger, J.G., G. Armilei, and M.C. Gorman, Transcription terminator-like element within a Saccharomyces cerevisiae promoter region. Mol Cell Biol, 1986. 6(4): p. 1095-101.

78. Flagfeldt, D.B., et al., Characterization of chromosomal integration sites for heterologous gene expression in Saccharomyces cerevisiae. Yeast, 2009. 26(10): p. 54551.

79. Dai, Z. and X. Dai, Nuclear colocalization of transcription factor target genes strengthens coregulation in yeast. Nucleic Acids Res, 2012. 40(1): p. 27-36.

80. Berger, A.B., et al., High-resolution statistical mapping reveals gene territories in live yeast. Nat Methods, 2008. 5(12): p. 1031-7.

81. Duan, Z., et al., A three-dimensional model of the yeast genome. Nature, 2010. 465(7296): p. 363-7.

82. Donczew, R. and S. Hahn, Mechanistic Differences in Transcription Initiation at TATALess and TATA-Containing Promoters. Mol Cell Biol, 2018. 38(1).

83. Verdone, L., et al., Chromatin remodeling during Saccharomyces cerevisiae ADH2 gene activation. Mol Cell Biol, 1996. 16(5): p. 1978-88.

84. Kalderon, D., et al., A short amino acid sequence able to specify nuclear location. Cell, 1984. 39(3 Pt 2): p. 499-509.

85. Dvir, S., et al., Deciphering the rules by which 5'-UTR sequences affect protein expression in yeast. Proc Natl Acad Sci U S A, 2013. 110(30): p. E2792-801.

86. Wang, M., S. Li, and H. Zhao, Design and engineering of intracellular-metabolitesensing/regulation gene circuits in Saccharomyces cerevisiae. Biotechnol Bioeng, 2016. 113(1): p. 206-15. 
87. Teo, W.S. and M.W. Chang, Bacterial XylRs and synthetic promoters function as genetically encoded xylose biosensors in Saccharomyces cerevisiae. Biotechnol J, 2015. 10(2): p. 315-22.

88. Stanton, B.C., et al., Genomic mining of prokaryotic repressors for orthogonal logic gates. Nat Chem Biol, 2014. 10(2): p. 99-105.

89. Brophy, J.A. and C.A. Voigt, Principles of genetic circuit design. Nat Methods, 2014. 11(5): p. 508-20.

90. Andrews, L.B., A.A.K. Nielsen, and C.A. Voigt, Cellular checkpoint control using programmable sequential logic. Science, 2018. 361(6408).

91. Auslander, D., et al., Programmable full-adder computations in communicating threedimensional cell cultures. Nat Methods, 2018. 15(1): p. 57-60.

92. Goentoro, L., et al., The incoherent feedforward loop can provide fold-change detection in gene regulation. Mol Cell, 2009. 36(5): p. 894-9.

93. Kuttykrishnan, S., et al., A quantitative model of glucose signaling in yeast reveals an incoherent feed forward loop leading to a specific, transient pulse of transcription. Proc Natl Acad Sci U S A, 2010. 107(38): p. 16743-8.

94. Sheff, M.A. and K.S. Thorn, Optimized cassettes for fluorescent protein tagging in Saccharomyces cerevisiae. Yeast, 2004. 21(8): p. 661-70.

95. Lam, A.J., et al., Improving FRET dynamic range with bright green and red fluorescent proteins. Nat Methods, 2012. 9(10): p. 1005-12.

96. Nagai, T., et al., A variant of yellow fluorescent protein with fast and efficient maturation for cell-biological applications. Nat Biotechnol, 2002. 20(1): p. 87-90.

97. Mumberg, D., R. Muller, and M. Funk, Yeast vectors for the controlled expression of heterologous proteins in different genetic backgrounds. Gene, 1995. 156(1): p. 119-22.

98. Sun, J., et al., Cloning and characterization of a panel of constitutive promoters for applications in pathway engineering in Saccharomyces cerevisiae. Biotechnology and Bioengineering, 2012. 109(8): p. 2082-2092.

99. Lubliner, S., L. Keren, and E. Segal, Sequence features of yeast and human core promoters that are predictive of maximal promoter activity. Nucleic Acids Research, 2013. 41(11): p. 5569-5581.

100. Blount, B.A., et al., Rational Diversification of a Promoter Providing Fine-Tuned Expression and Orthogonal Regulation for Synthetic Biology. Plos One, 2012. 7(3).

101. Guldener, U., et al., A new efficient gene disruption cassette for repeated use in budding yeast. Nucleic Acids Res, 1996. 24(13): p. 2519-24.

102. Goldstein, A.L., X. Pan, and J.H. McCusker, Heterologous URA3MX cassettes for gene replacement in Saccharomyces cerevisiae. Yeast, 1999. 15(6): p. 507-11.

103. Holstege, F.C., et al., Dissecting the regulatory circuitry of a eukaryotic genome. Cell, 1998. 95(5): p. 717-28.

104. Wapinski, I., et al., Natural history and evolutionary principles of gene duplication in fungi. Nature, 2007. 449(7158): p. 54-61.

105. Kaplan, N., et al., The DNA-encoded nucleosome organization of a eukaryotic genome. Nature, 2009. 458(7236): p. 362-6.

106. Bilitchenko, L., et al., Eugene--a domain specific language for specifying and constraining synthetic biological parts, devices, and systems. PLoS One, 2011. 6(4): p. e18882. 
Figure 1

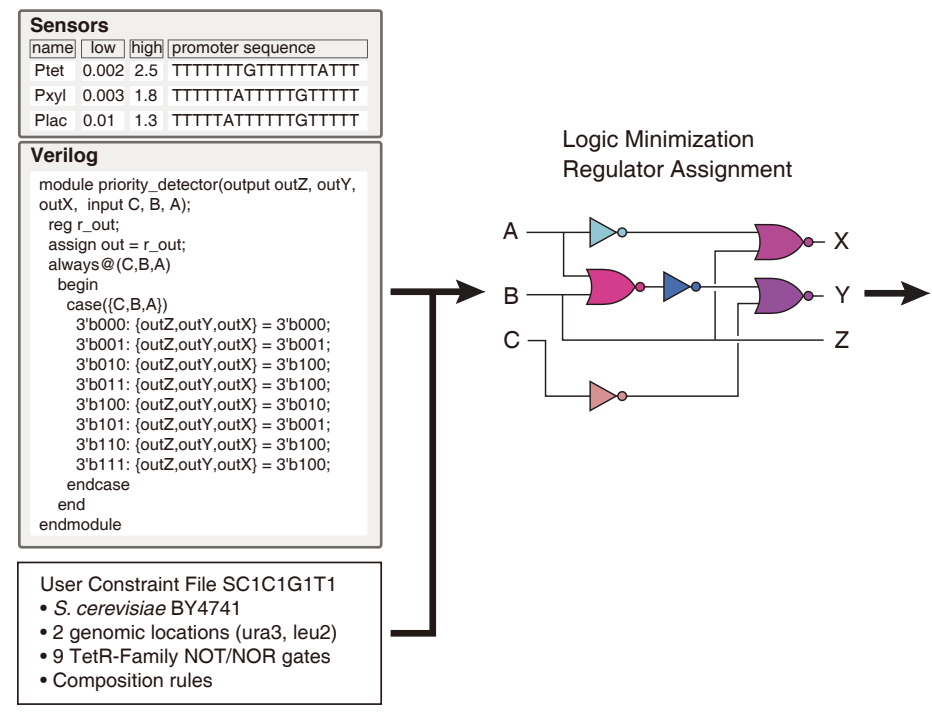

Map Circuit to DNA

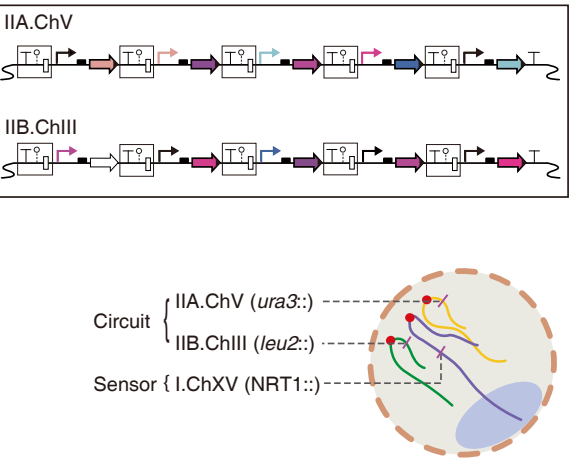


Figure 2

a

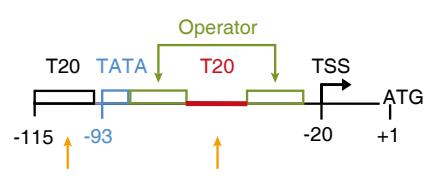

Mutations to suppress recombination

b

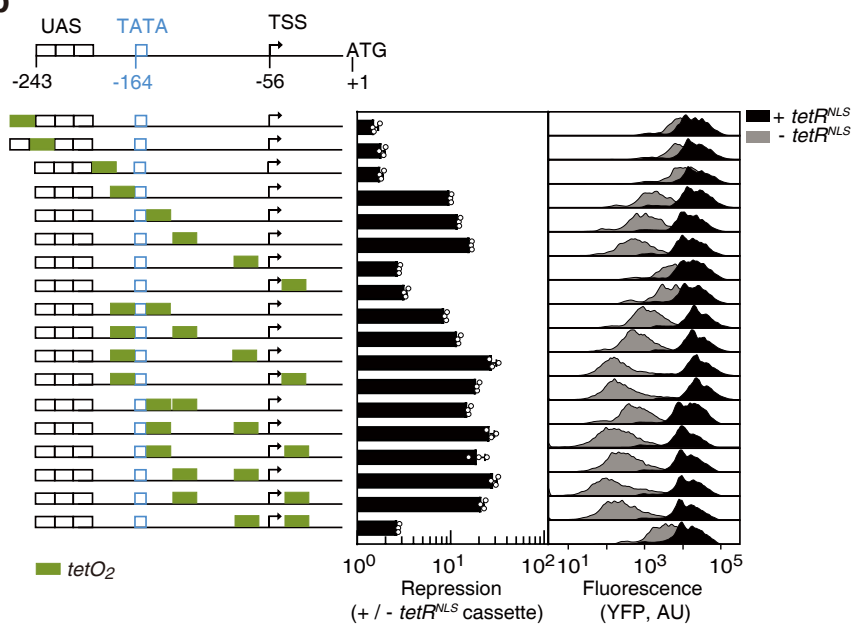

C

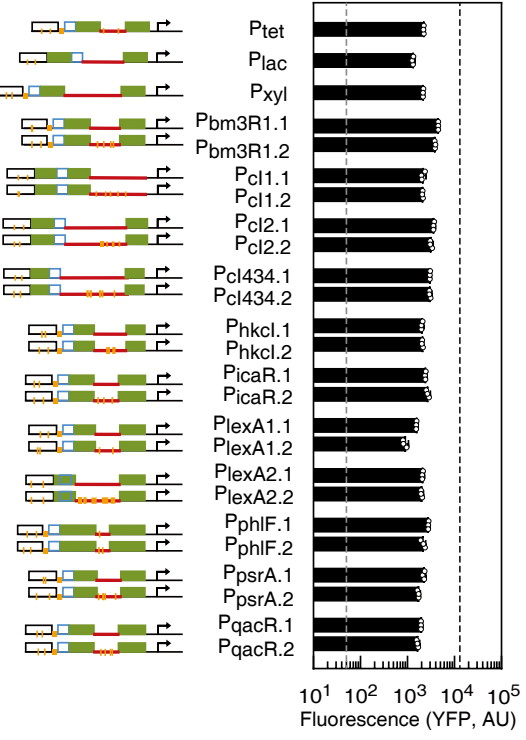


Figure 3

a

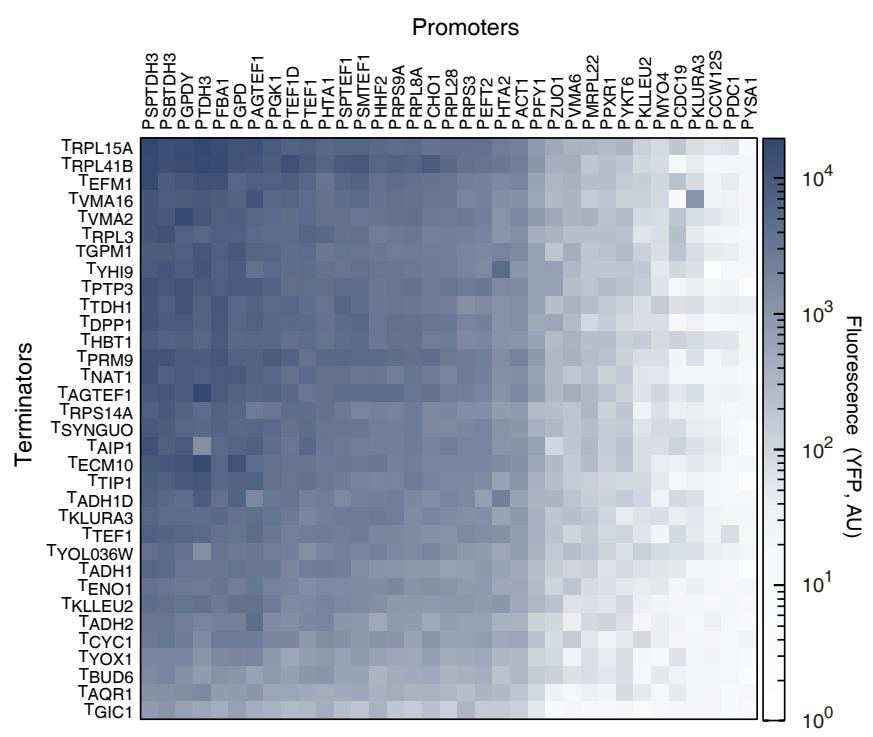

b
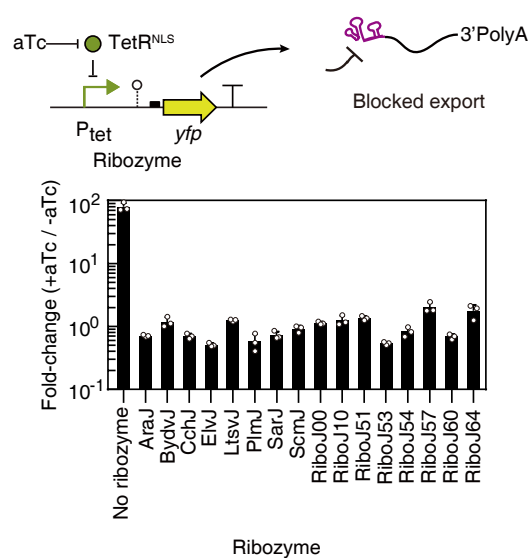

c
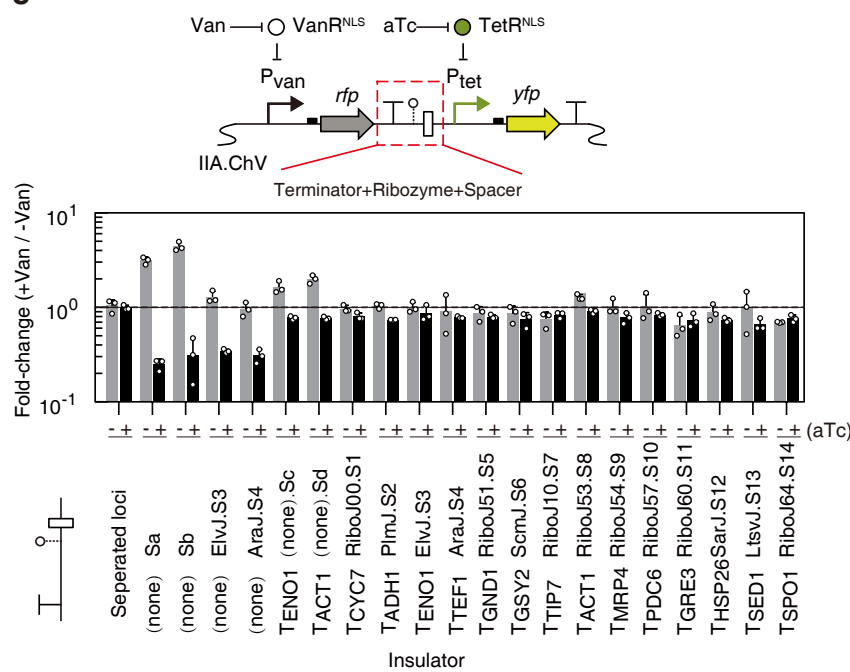
Figure 4

a

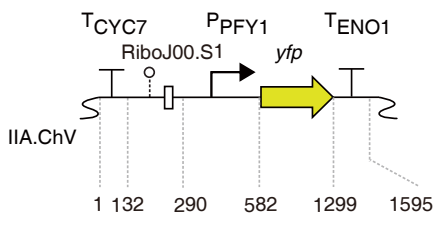

c
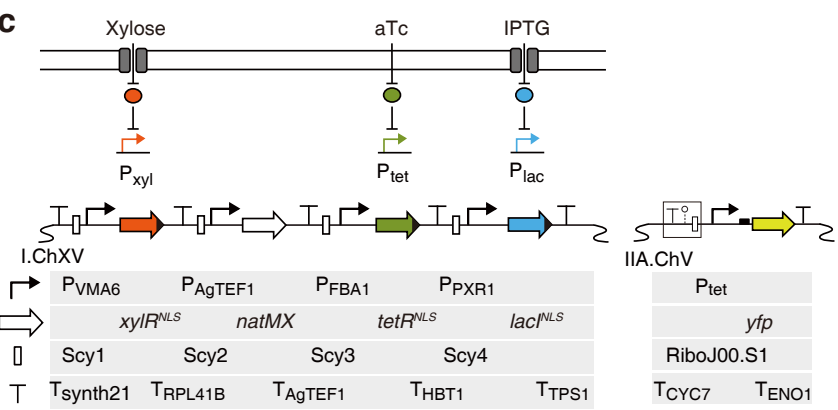

d

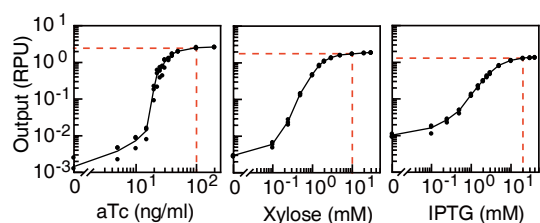

e

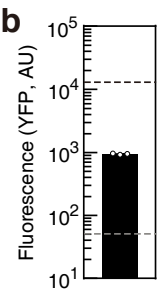

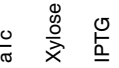

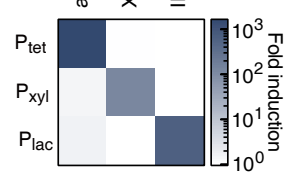


Figure 5

a
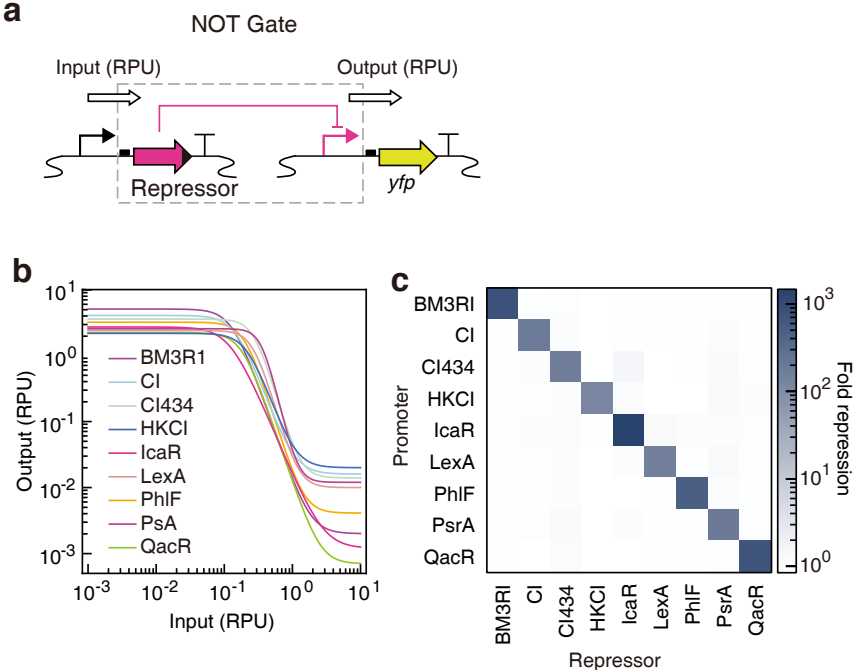

d

e

Repressor

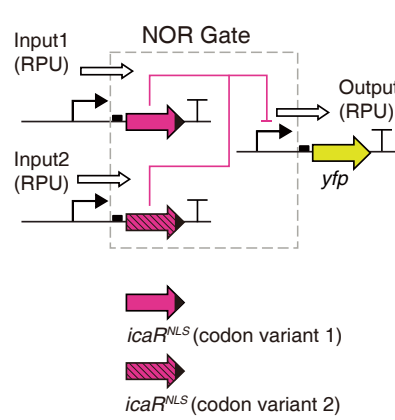

e Same chromosome

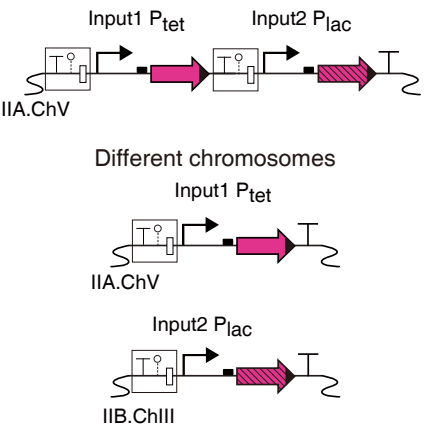

f

g
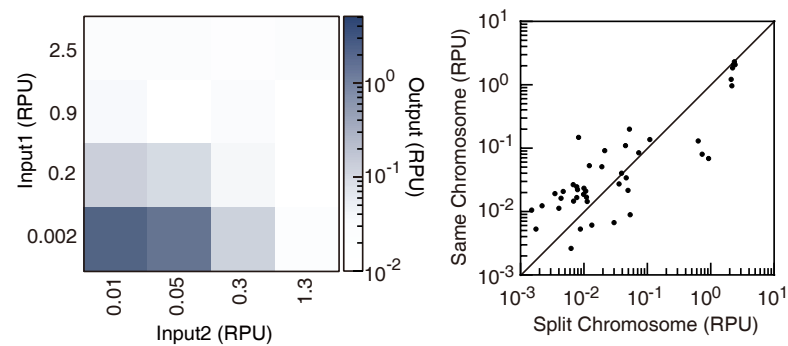

h

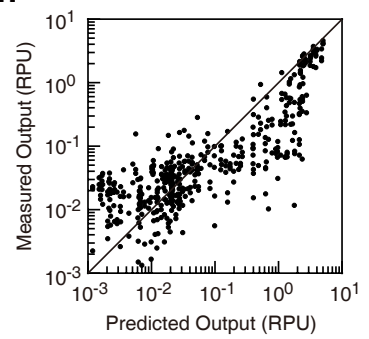


Figure 6

a

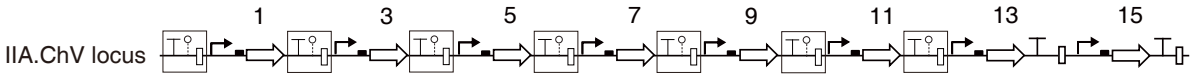

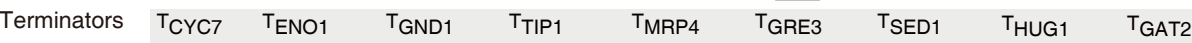
Spacers RiboJ00.S1 ElvJ.S3 RiboJ51.S5 RiboJ10.S7 RiboJ54.S9 RiboJ60.S11 LtsvJ.S13 $\quad$ S15 $\quad$ S17

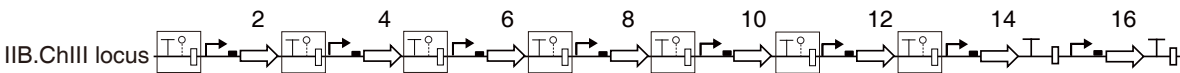

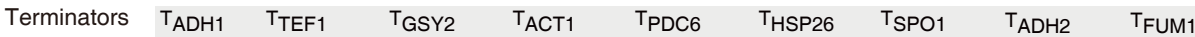
$\begin{array}{llllllll}\text { Spacers } & \text { PImJ.S2 AraJ.S4 } & \text { ScmJ.S6 } & \text { RiboJ53.S8 } & \text { RiboJ57.S10 SarJ.S12 } & \text { RiboJ64.S14 } & \text { S16 } & \text { S18 }\end{array}$

C

\section{0x06}
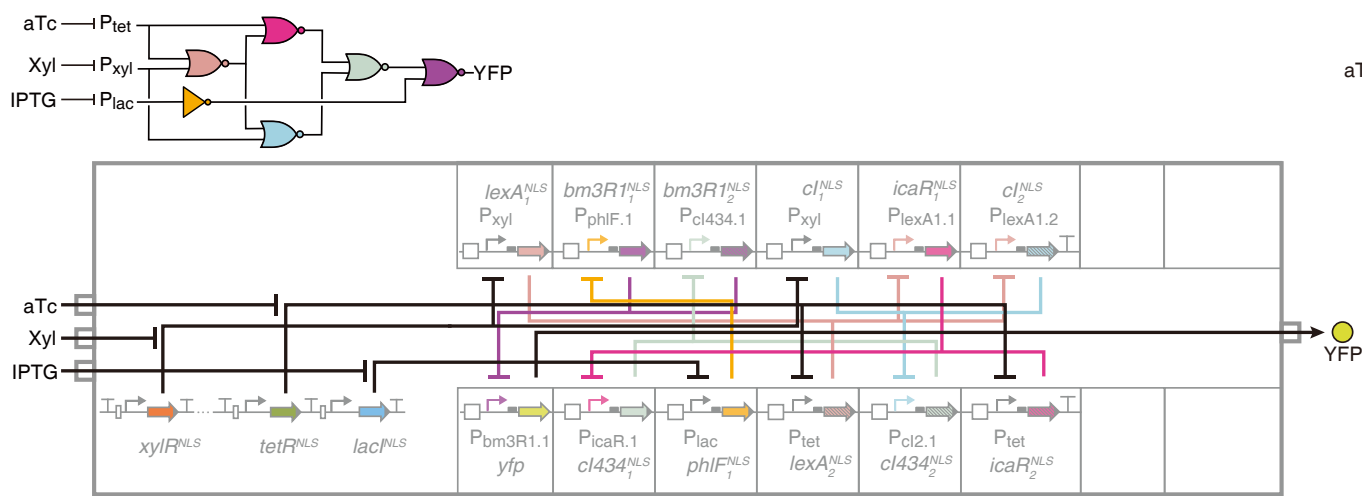

$0 \times 6 B$
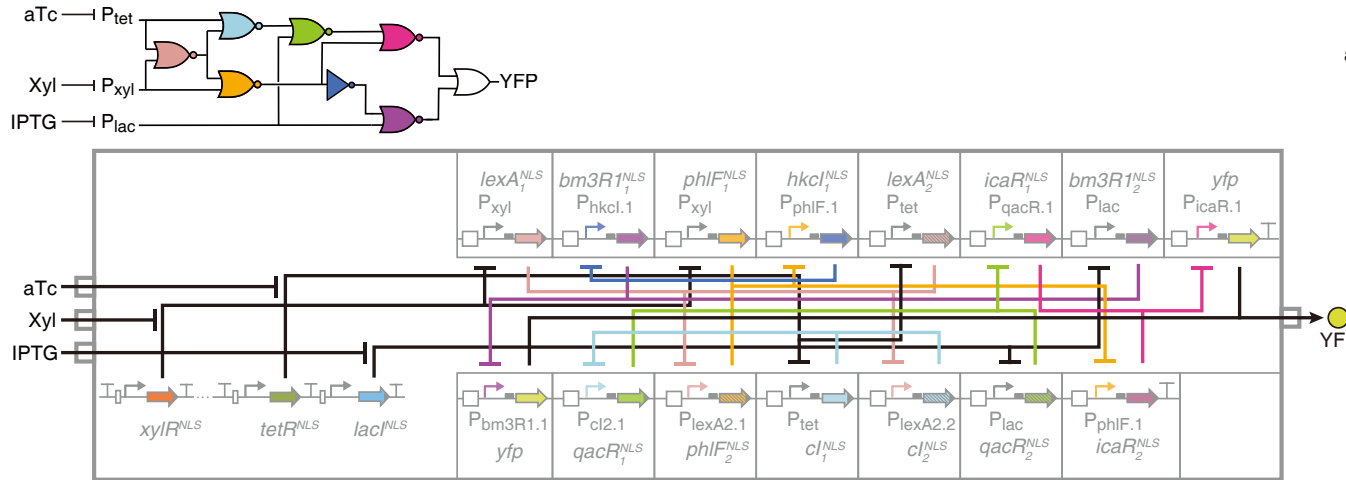

0X9E
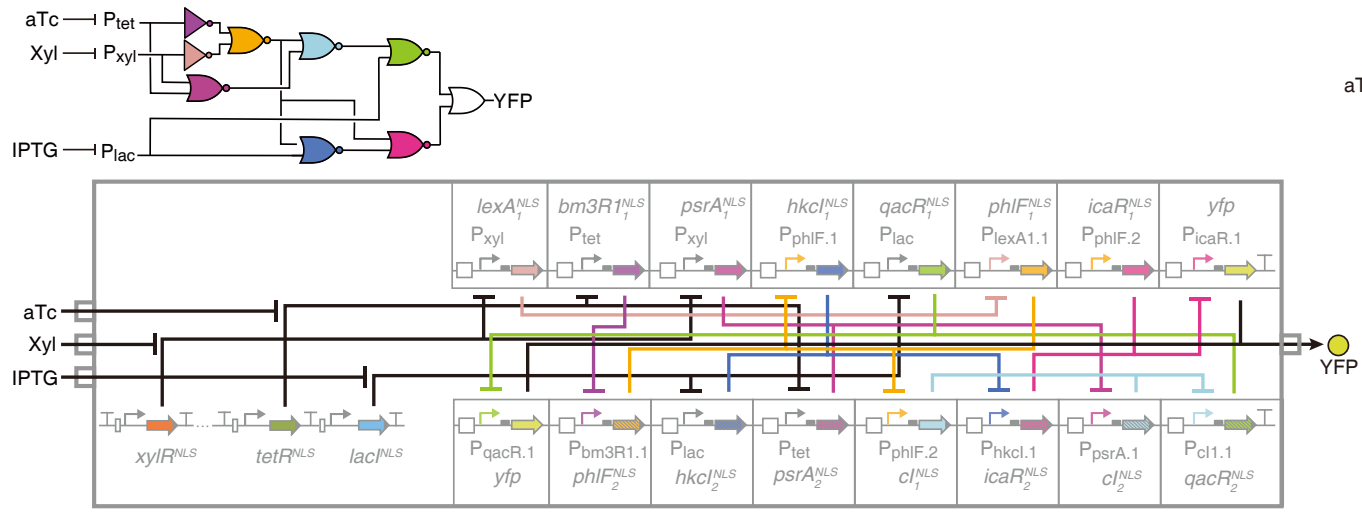

b

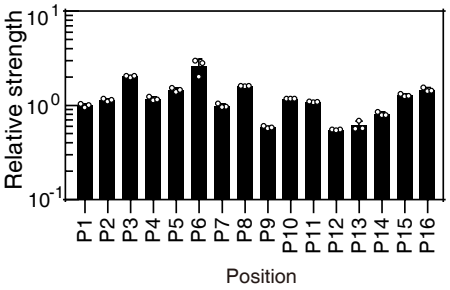

aTc / Xyl / IPTG

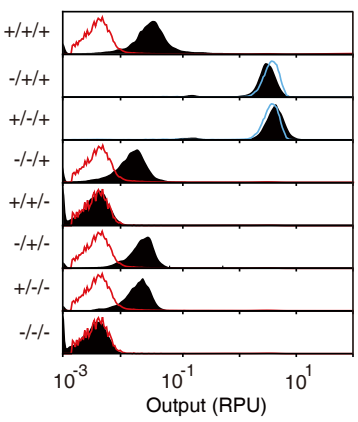

aTc / Xyl / IPTG

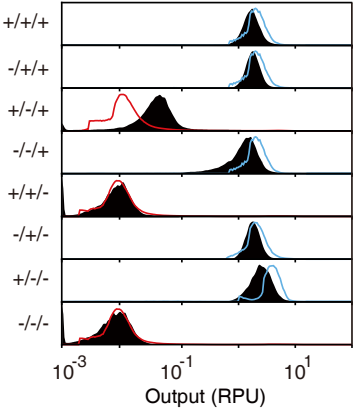

aTc / Xyl / IPTG

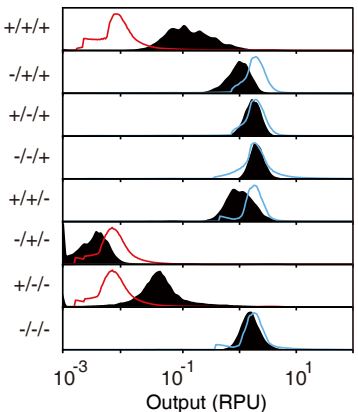


Figure 6
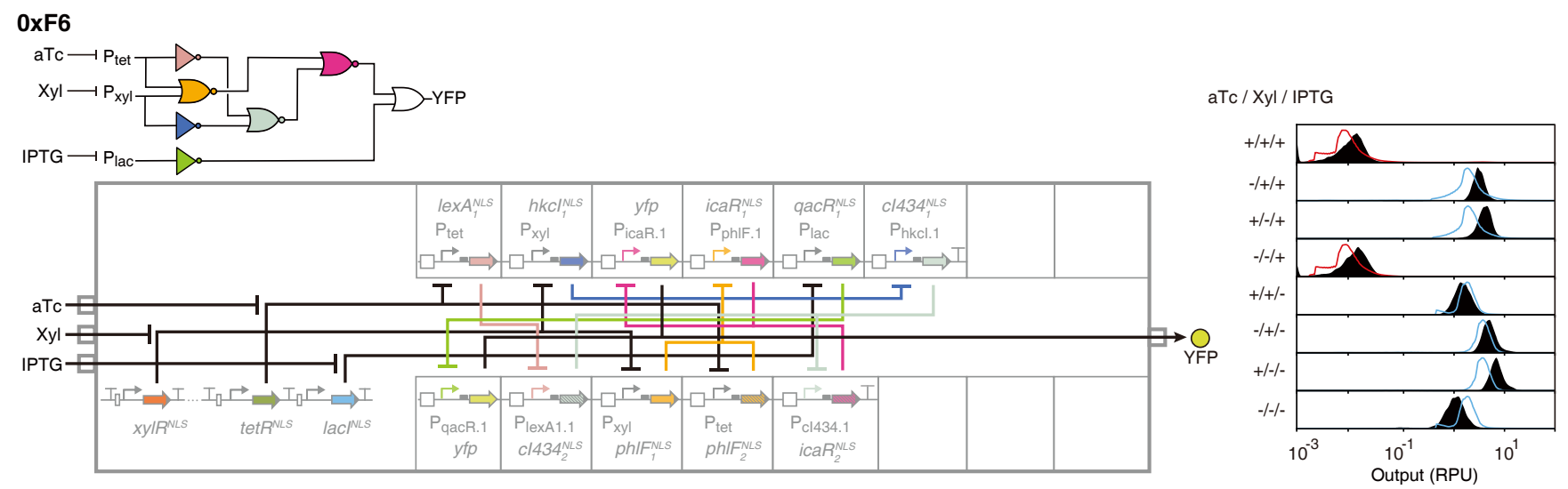

0xA1
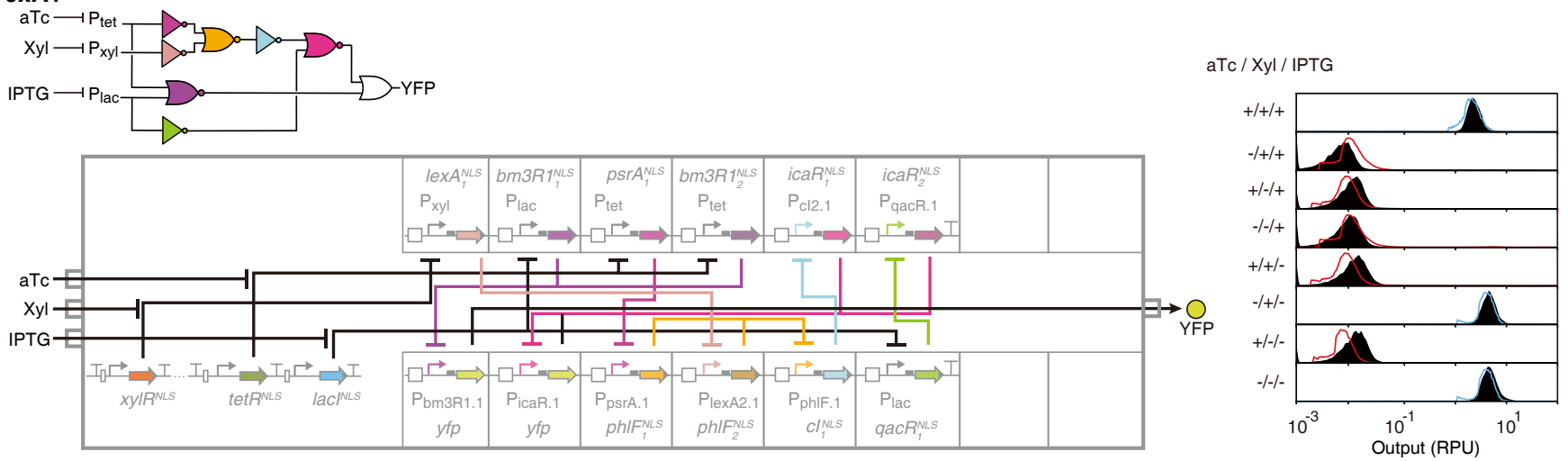
Figure 7

a

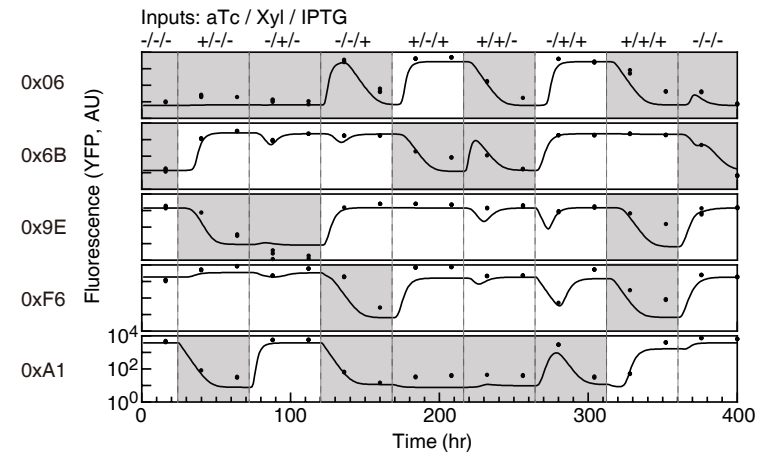

b

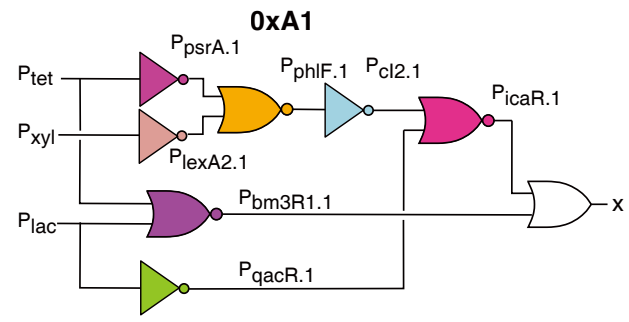

C

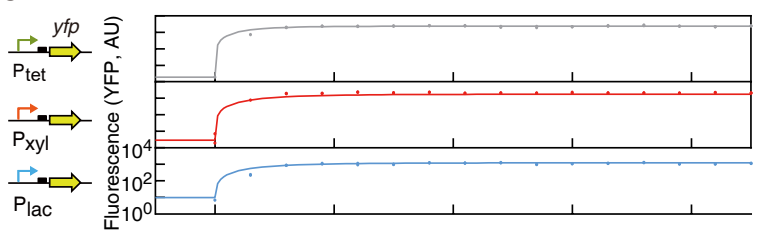

$\underset{\text { PsrA.1 }}{\stackrel{r f p}{\rightleftarrows}}$

$\stackrel{P \text { psrA.1 }}{\longrightarrow}$

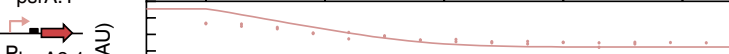

$\stackrel{P \text { lexA2.1 }}{\longrightarrow}$ 品

PqacR.1

$\overrightarrow{P \text { phIF.1 }}$

$\underset{\mathrm{P}_{\mathrm{Cl} 2.1} \Rightarrow}{\longrightarrow}$

$\vec{r}$

Pbm3R1.1

$\overrightarrow{\vec{r}} \overrightarrow{\mathrm{P}}$

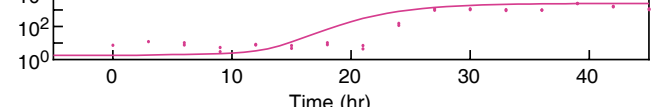

d

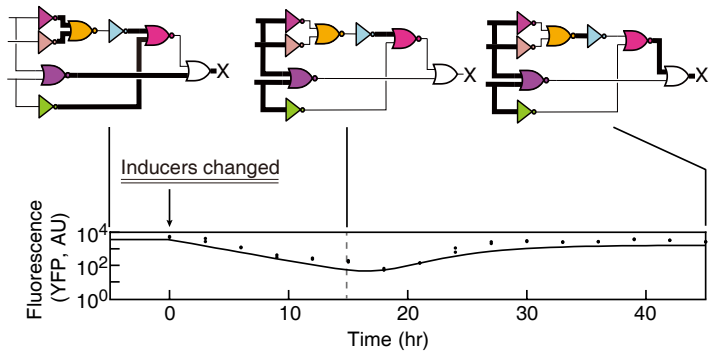

